\title{
Hydrostatic models for the rotation of extra-planar gas in disk galaxies $\star$
}

\author{
M. Barnabè ${ }^{1,2}$, L. Ciotti ${ }^{2}$, F. Fraternali ${ }^{3}$, and R. Sancisi ${ }^{1,4}$ \\ 1 Kapteyn Astronomical Institute, University of Groningen, PO Box 800, 9700 AV Groningen, The Netherlands \\ e-mail: M.Barnabe@astro.rug.nl \\ 2 Dipartimento di Astronomia, Università di Bologna, via Ranzani 1, 40127 Bologna, Italy \\ 3 Theoretical Physics, University of Oxford, 1 Keble Road, Oxford, OX1 3NP, UK \\ ${ }^{4}$ INAF-Osservatorio Astronomico di Bologna, via Ranzani 1, 40127 Bologna, Italy
}

Received 9 May 2005 / Accepted 25 August 2005

\section{ABSTRACT}

We show that fluid stationary models are able to reproduce the observed negative vertical gradient of the rotation velocity of the extra-planar gas in spiral galaxies. We have constructed models based on the simple condition that the pressure of the medium does not depend on density alone (baroclinic instead of barotropic solutions: isodensity and isothermal surfaces do not coincide). As an illustration, we have successfully applied our method to reproduce the observed velocity gradient of the lagging gaseous halo of NGC 891. The fluid stationary models discussed here can describe a hot homogeneous medium as well as a "gas" made of discrete, cold H I clouds with an isotropic velocity dispersion distribution. Although the method presented here generates a density and velocity field consistent with observational constraints, the stability of these configurations remains unresolved.

Key words. galaxies: general - galaxies: halo - galaxies: individual: NGC 891 - galaxies: kinematics and dynamics - galaxies: structure ISM: kinematics and dynamics

\section{Introduction}

Observations at various wavelengths show that some spiral galaxies are surrounded by a gaseous halo. This extra-planar gas is multiphasic: it is detected in H I (e.g., Swaters et al. 1997), H $\alpha$ (Rand 2000; Rossa et al. 2004) and X-ray observations (Wang et al. 2001; Strickland et al. 2004). In particular, high-sensitivity H I observations of edge-on galaxies like NGC 891 (Swaters et al. 1997; Fraternali et al. 2004a) and UGC 7321 (Matthews \& Wood 2003) reveal neutral gas emission up to large distances from the plane (e.g. see the H I map of NGC 891 in Fraternali et al. 2005) and the presence of a negative vertical gradient in the gas rotational velocity. A similar decrease of the rotational velocity with distance from the plane has also been observed in the diffuse ionized gas halo of NGC 891 (Rand 1997) and of NGC 5775 (Rand 2000).

The two major issues regarding the extra-planar gas are those of its origin and of its dynamical state. These are closely related. For example, the halo gas could be the result of cosmological accretion (e.g., see Binney 2005), or of a galactic fountain (Shapiro \& Field 1976; Bregman 1980), or both. Different structures and kinematics would be expected for these cases.

\footnotetext{
* Appendices are only available in electronic form at
} http://www. edpsciences.org
Thus, the study of the origin and dynamics of the extra-planar gas not only is important in itself, but may also provide new insight into the formation and the structure of spiral galaxies. Furthermore, it may bring new evidence for the vertical distribution of dark matter. In this paper we focus on the problem of the dynamical state of extra-planar gas.

Two "extreme" types of models have been considered for the extra-planar gas and in particular for its decreasing rotational velocity: the ballistic and the fluid homogeneous models.

Ballistic models describe the gas as an inhomogeneous collection of clouds, subject only to the gravitational potential of the galaxy: for example, in the galactic fountain model ionized gas is ejected from the galactic disk due to stellar winds and supernova explosions, and then cools and falls back ballistically (Bregman 1980). These models are able to explain vertical motions of the cold ( $\mathrm{HI})$ and warm $(\mathrm{H} \alpha)$ gas components observed in several spiral galaxies (e.g. Fraternali et al. 2004b; Boomsma et al. 2005). However, Collins et al. (2002) have tried a ballistic model to describe the extra-planar ionised gas of NGC 891 and found problems in reproducing the observed kinematics. These authors suggest that the discrepancy could be solved by considering "the presence of drag between disk and halo, such as through magnetic tension or viscous interactions between 
clouds. Alternatively, an outwardly directed pressure gradient could explain the gas kinematics".

The concept of pressure in the physics of the interstellar medium is a complex one, as there are several contributing sources of pressure, e.g. thermal, kinetic (or turbulent), magnetic, cosmic ray and radiation (e.g., see Boulares \& Cox 1990 , and references therein). For simplicity, in the following we will restrict our discussion to thermal and kinetic pressure. In fluid homogeneous models, the extra-planar gas is described as a stationary rotating fluid without any motion along the radial and vertical directions, with the galaxy gravitational field balanced by the pressure gradient and the centrifugal force. This approach has not been fully explored in all its possibilities, and only a few attempts have been made (e.g., see Benjamin 2002). Here we extend a preliminary analysis of fluid homogeneous stationary models (Barnabè et al. 2005) to explain the vertical gradient of rotational velocity observed in extra-planar gas. In particular, we show that models in which the gas pressure does not depend on the density alone (baroclinic configurations) are able to reproduce the observed vertical gradient. In addition, we show that baroclinic solutions could provide the drag invoked by Collins et al. (2002). This suggests that a correct description of the extra-planar gas dynamics may be found in "hybrid" ballistic-fluid stationary models. Finally, we address the question of the physical interpretation of our solutions and suggest, as an alternative to the hypothesis of a hot homogeneous medium, the possibility of a "gas" of cold $\mathrm{H}$ I clouds described by the stationary (fluid) Jeans equations, with the sustaining pressure given by a globally isotropic velocity dispersion tensor.

The paper is organized as follows: in Sects. 2 and 3, we briefly introduce the baroclinic solutions and derive simple and general rules for their construction. In Sect. 4 we construct a fluid homogeneous and stationary model for the galaxy NGC 891, and in Sect. 5 we discuss the results from an astrophysical point of view. In the Appendices a simple, fully analytical model of a gas distribution with low rotation at high $z$ is presented, together with the numerical code adopted for the case of NGC 891.

\section{The fluid approach}

\subsection{The fluid equations}

We consider a gaseous axisymmetric system in permanent rotation, under the influence of an axisymmetric gravitational potential $\Phi_{\text {tot }}(R, z)$ : because of the axial symmetry, all the physical variables depend only on the cylindrical coordinates $R$ and $z$. The stationary hydrodynamical equations for the gas are then

$$
\left\{\begin{array}{l}
\frac{1}{\rho} \frac{\partial P}{\partial z}=-\frac{\partial \Phi_{\mathrm{tot}}}{\partial z}, \\
\frac{1}{\rho} \frac{\partial P}{\partial R}=-\frac{\partial \Phi_{\mathrm{tot}}}{\partial R}+\Omega^{2} R,
\end{array}\right.
$$

where $\rho, P$ and $\Omega$ denote the gas density, pressure and angular velocity, respectively; the gas rotational velocity is given by $v_{\varphi}=\Omega R$, while $v_{R}=v_{z}=0$. Note that $\Phi_{\text {tot }}$ represents the total gravitational potential, including the gas contribution. Later on, we will assume the condition that the gas is not self-gravitating and therefore $\Phi_{\text {tot }}=\Phi$, where the galaxy gravitational potential $\Phi$ is the sum of the dark halo and the stellar disk potentials.

In standard applications, as for example the setting up of initial conditions for hydrodynamical simulations, the above equations are solved adopting a barotropic pressure distribution and neglecting the gas self-gravity. Thus, one fixes the gravitational potential $\Phi$ and a specific function $P(\rho)$, and integrates the first of Eqs. (1) with the boundary $\rho(R, 0)$ or imposing $\rho(R, \infty)=0$. The angular velocity $\Omega$ is obtained from the second of Eqs. (1). This leads to cylindrical rotation, i.e. $\Omega=\Omega(R)$. According to the Poincaré-Wavre theorem (Lebovitz 1967; Tassoul 1980), cylindrical rotation is equivalent to the fact that the acceleration field at the r.h.s. of Eqs. (1) can be obtained from an effective potential $\Phi_{\text {eff }}$ (see Eq. (8)), or that the gas density and pressure are stratified on $\Phi_{\text {eff }}$, and so the fluid is barotropic.

Cylindrical rotation is in disagreement with the observed vertical gradient of the extra-planar gas rotation velocity, and this would seem to argue against the applicability of fluid stationary models. However, in the next section we will show that it is possible to construct baroclinic equilibrium solutions with a negative velocity gradient along $z$. Baroclinic solutions have been studied in the past for problems ranging from geophysics to the theory of sunspots and to galactic dynamics ${ }^{1}$ (e.g., see Rosseland 1926; Tassoul 1980; Waxman 1978, and references therein).

\subsection{Baroclinic solutions}

For simplicity we restrict ourselves to non self-gravitating gas distributions, even though several results hold also in the selfgravitating case. We fix the total gravitational potential $\Phi(R, z)$ for the galaxy but, at variance with the standard treatment, we prescribe a density distribution $\rho(R, z)$ for the gas vanishing at infinity. (We do not address here the much more difficult problem of a consistent assignement of non-zero pressure as a boundary condition.) The first of Eqs. (1) is integrated for the pressure as

$P(R, z)=\int_{z}^{\infty} \rho \frac{\partial \Phi}{\partial z^{\prime}} \mathrm{d} z^{\prime}$,

where we also assume $P(R, \infty)=0$. In general, the obtained pressure $P$ (and the corresponding temperature $T=\mu m_{\mathrm{p}} P / \mathrm{k} \rho$ ) cannot be expressed as a function of $\rho$ only, and so the system is baroclinic. Accordingly, the rotational velocity field

$v_{\varphi}^{2}(R, z)=\frac{R}{\rho} \frac{\partial P}{\partial R}+R \frac{\partial \Phi}{\partial R}$

depends both on $R$ and $z$. The major problem posed by the construction of baroclinic solutions is the fact that, for an arbitrary choice of $\rho$ and $\Phi$, the existence of physically acceptable solutions (i.e. configurations for which $v_{\varphi}^{2} \geq 0$ everywhere) is not

\footnotetext{
${ }^{1}$ In particular, isotropic axisymmetric galaxy models can be interpreted as baroclinic fluid configurations, and show streaming velocities often decreasing with $z$ (e.g., see Lanzoni \& Ciotti 2003, and Ciotti \& Bertin 2005 for simple examples).
} 
guaranteed. Due to the arbitrariness of the chosen density field, a negative radial pressure gradient in Eq. (3) can be dominant for some values of $R$ and $z$. Thus, before addressing the specific case of the extra-planar gas in NGC 891, in the next section we present a few mathematical results that will be used as guidelines in the construction of physically acceptable baroclinic solutions. In Appendix A we present a fully analytical, baroclinic model as an example of the procedure described above.

\section{Simple families of solutions}

The starting point of the following analysis is obtained by combining Eqs. (2) and (3) and integrating by parts with the assumption that $P$ and $\rho \partial \Phi / \partial R$ vanish for $z=\infty$, thus obtaining the exact relation

$\frac{\rho v_{\varphi}^{2}}{R}=\int_{z}^{\infty}\left(\frac{\partial \rho}{\partial R} \frac{\partial \Phi}{\partial z^{\prime}}-\frac{\partial \rho}{\partial z^{\prime}} \frac{\partial \Phi}{\partial R}\right) \mathrm{d} z^{\prime} \equiv \mathrm{C}[\rho, \Phi]$.

This "commutator-like" relation is not new (e.g., see Rosseland 1926, Waxman 1978 and, in the context of stellar dynamics, Hunter 1977); here we note that the positivity of the integrand in Eq. (4) is a sufficient (but not necessary) condition to obtain $v_{\varphi}^{2} \geq 0$ everywhere. Therefore, physically acceptable solutions are obtained if one assume a potential $\Phi$ for which $\partial \Phi / \partial R \geq 0$ and $\partial \Phi / \partial z \geq 0$ (the usual situation) and a density distribution so that $\partial \rho / \partial z \leq 0$ and $\partial \rho / \partial R \geq 0$.

In addition, the bilinearity of $\mathrm{C}[\rho, \Phi]$ can be used to construct more complicate solutions starting from simple, physically acceptable "building block" configurations. From Eq. (4) it follows that the rotational velocity associated with $\rho=$ $\rho_{1}+\rho_{2}$ is

$v_{\varphi}^{2}=\frac{\rho_{1} v_{\varphi, 1}^{2}+\rho_{2} v_{\varphi, 2}^{2}}{\rho_{1}+\rho_{2}}$,

where $\rho_{1} v_{\varphi, 1}^{2} / R \equiv \mathrm{C}\left[\rho_{1}, \Phi\right]$ and $\rho_{2} v_{\varphi, 2}^{2} / R \equiv \mathrm{C}\left[\rho_{2}, \Phi\right]$. Also, if $\Phi=\Phi_{1}+\Phi_{2}$, then

$v_{\varphi}^{2}=v_{\varphi, 1}^{2}+v_{\varphi, 2}^{2}$.

\subsection{Gas density distributions with a factor stratified on the effective potential}

We now elaborate in more detail the general results of Eqs. (4)-(6). Let us consider the factorized gas density distribution

$\rho(R, z)=h(R, z) \rho_{\mathrm{e}}\left(\Phi_{\mathrm{eff}}\right)$,

where $h$ is a non negative function and

$\Phi_{\text {eff }} \equiv \Phi-\int_{R_{0}}^{R} \Omega^{2}\left(R^{\prime}\right) R^{\prime} \mathrm{d} R^{\prime}$

is the effective potential associated with the total potential $\Phi$ and with a prescribed cylindrical rotation law $\Omega(R) ; R_{0}$ is an arbitrary but fixed radius. We assume that $\rho_{\mathrm{e}}$ in Eq. (7) is a solution of the equation

$\nabla P=-\rho \nabla \Phi_{\mathrm{eff}}$ with assigned $P=P(\rho)$. For $\Omega=0$ one obtains a hydrostatic (and therefore barotropic) solution in the potential $\Phi$ (that we indicate with $\rho_{\mathrm{h}}$ ), while for $\Omega(R) \neq 0$ one has a cylindrical rotation (barotropic) solution.

In practice, by adopting factorization (7) one modifies a gas distribution with a cylindrical velocity field: this approach is of obvious interest because families of $\rho_{\mathrm{e}}\left(\Phi_{\text {eff }}\right)$ can be easily constructed (see Appendix B). Substituting Eq. (7) in Eq. (4) gives

$$
\begin{aligned}
\frac{\rho v_{\varphi}^{2}}{R}= & \int_{z}^{\infty}\left(\frac{\partial h}{\partial R} \frac{\partial \Phi}{\partial z^{\prime}}-\frac{\partial h}{\partial z^{\prime}} \frac{\partial \Phi}{\partial R}\right) \rho_{\mathrm{e}} \mathrm{d} z^{\prime} \\
& -R \Omega^{2}(R) \int_{z}^{\infty} h \frac{\partial \rho_{\mathrm{e}}}{\partial z^{\prime}} \mathrm{d} z^{\prime}
\end{aligned}
$$

Equations (5) and (10) prove the existence of physically acceptable baroclinic solutions. For example, $\rho=\rho_{\mathrm{h}}(\Phi)+\rho_{\mathrm{e}}\left(\Phi_{\mathrm{eff}}\right)$ leads to

$v_{\varphi}^{2}=\frac{\rho_{\mathrm{e}} R^{2} \Omega^{2}(R)}{\rho_{\mathrm{h}}+\rho_{\mathrm{e}}}$.

Another case of physically acceptable solutions is $\rho=$ $A(R) \rho_{\mathrm{h}}(\Phi)$, with $A(R)$ increasing and approaching a constant value for $R \gg 1$. In this case $v_{\varphi}^{2}$ decreases reaching systemic (zero) velocity at infinity ${ }^{2}$.

We now apply the method we have just described to more specific astrophysically relevant cases: a homeoidally stratified potential and a razor-thin uniform disk. For simplicity, we assume in Eq. (7) a hydrostatic density factor $\rho_{\mathrm{h}}(\Phi)$.

\subsubsection{Homeoidal potential}

Let $\Phi(\ell)$ be an homeoidally stratified potential with $\ell^{2} \equiv$ $R^{2}+z^{2} / q_{\Phi}^{2}$ and $0<q_{\Phi} \leq 1$; two well-known examples are the Binney (1981) logarithmic potential and the Evans (1994) spheroidal potentials. We write Eq. (7) as

$\rho(R, z)=A(R) B(m) \rho_{\mathrm{h}}(\Phi)$,

where $m^{2} \equiv R^{2}+z^{2} / q_{\mathrm{g}}^{2}$ and $0<q_{\mathrm{g}} \leq 1 ; A(R)$ and $B(m)$ are positive functions. From Eq. (10) we have

$$
\begin{aligned}
\frac{\rho v_{\varphi}^{2}}{R}= & \left(\frac{1}{q_{\Phi}^{2}}-\frac{1}{q_{\mathrm{g}}^{2}}\right) A(R) R \int_{z}^{\infty} \frac{B^{\prime}(m)}{m} \rho_{\mathrm{h}}(\Phi) \Phi^{\prime}(\ell) \frac{z^{\prime}}{\ell} \mathrm{d} z^{\prime} \\
& +\frac{A^{\prime}(R)}{q_{\Phi}^{2}} \int_{z}^{\infty} B(m) \rho_{\mathrm{h}}(\Phi) \Phi^{\prime}(\ell) \frac{z^{\prime}}{\ell} \mathrm{d} z^{\prime},
\end{aligned}
$$

and so $v_{\varphi}^{2} \geq 0$ if

$\Phi^{\prime}(\ell) \geq 0, \quad A^{\prime}(R) \geq 0, \quad B^{\prime}(m) \leq 0, \quad q_{\mathrm{g}} \leq q_{\Phi}$.

Note that $\rho_{\mathrm{h}}$ does not enter in the sufficient condition (14). The condition on flattenings $\left(q_{\mathrm{g}} \leq q_{\Phi}\right)$ requires that the gas density distribution must be stratified on homeoids which are flatter than the isopotential surfaces. This is, therefore, always satisfied for a flat gas distribution in the spherically symmetric monopole-dominated far field of any finite mass system (e.g. a stellar disk).

\footnotetext{
${ }^{2}$ E.g., the function $A(R)=R /(1+R)$.
} 


\subsubsection{Razor-thin uniform disk}

We now discuss the case of a razor-thin uniform disk. What are the conditions for having physically acceptable $\left(v_{\varphi}^{2}>0\right)$ solutions near the disk? We explore this issue by assuming $\rho=$ $A(R) \rho_{\mathrm{h}}(\Phi)$ in Eq. (7) (or $B=1$ in Eq. (12)) and

$\Phi=2 \pi G \Sigma_{0} z$.

From Eq. (10) (or Eq. (13))

$\frac{\rho_{\mathrm{h}} v_{\varphi}^{2}}{R}=2 \pi G \Sigma_{0} \frac{A^{\prime}(R)}{A(R)} \int_{z}^{\infty} \rho_{\mathrm{h}}(\Phi) \mathrm{d} z^{\prime}$,

i.e. $A^{\prime}(R) \geq 0$ is the necessary and sufficient condition to have $v_{\varphi}^{2} \geq 0$ in such a case.

The physical reason for this condition is that a gas distribution $\rho(R, z)$ not stratified on $\Phi$ as given by Eq. (15) must be rotating and according to Eq. (3) its pressure must be radially increasing. From Eqs. (15) and (2) it follows that in the present case the pressure is proportional to the gas column density. This means that, in a vertical gravitational field, $v_{\varphi}^{2} \geq 0$ whenever the column density is radially increasing. Note that this trend is consistent with the radial $\mathrm{H}$ I density distribution observed in several spiral galaxies (see Cayatte et al. 1994). Obviously, the case presented by more realistic disks (as the exponential disk in Sect. 4) will require the explicit construction of the whole equilibrium solution to check for the positivity of $v_{\varphi}^{2}$.

\section{Application to NGC 891}

We now apply the general results of Sect. 3 to the modeling of the extra-planar gas of NGC 891 in an attempt to reproduce its major features and in particular the decline of the rotational velocity with increasing $z$. The rotational velocity field resulting from this modeling will be compared to the $\mathrm{H}$ I rotation curves derived by Fraternali et al. (2005).

\subsection{The galaxy model}

We consider a very idealized mass model for NGC 891, consisting of three components: an exponential stellar disk, a dark matter halo with an asymptotically flat rotation curve, and a centrally peaked density distribution. This model provides the gravitational potential supporting a (non self-gravitating) baroclinic gas distribution. Its parameters are fixed to reproduce the observed rotation curve in the galactic plane and to obey other observational constraints specified in the following.

The surface density of the stellar disk is

$\Sigma(R)=\frac{M_{\mathrm{d}}}{2 \pi R_{\mathrm{d}}^{2}} \mathrm{e}^{-R / R_{\mathrm{d}}}$,

where $M_{\mathrm{d}}$ and $R_{\mathrm{d}}$ are the disk mass and scale-length, respectively. Its gravitational potential is given by

$\Phi_{\mathrm{d}}(R, z)=-\frac{G M_{\mathrm{d}}}{R_{\mathrm{d}}} \int_{0}^{\infty} \frac{J_{0}(k \tilde{R}) \mathrm{e}^{-k|\tilde{z}|}}{\left(1+k^{2}\right)^{3 / 2}} \mathrm{~d} k$,

where $J_{0}$ is the zeroth-order Bessel function of the first kind, and $\tilde{R} \equiv R / R_{\mathrm{d}}, \tilde{z} \equiv z / R_{\mathrm{d}}$ (e.g. Binney \& Tremaine 1987).

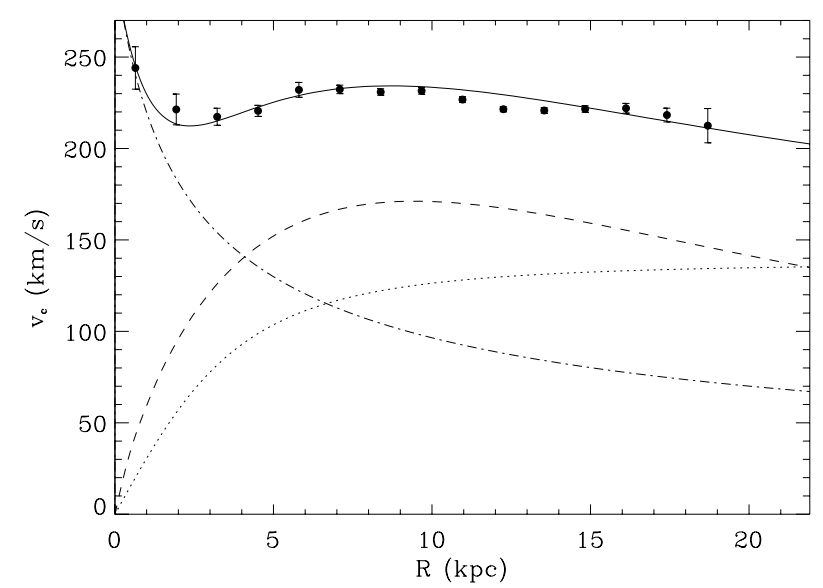

Fig. 1. The adopted mass decomposition for NGC 891. Solid dots represent the observed $\mathrm{H}$ I rotation curve (Fraternali et al. 2005), and the solid line the model rotation curve. The dashed, dotted, and dot-dashed lines show the separate contributions of the disk, the dark matter halo, and of the spherical Jaffe mass component, respectively.

The observed rotation curve is reproduced by adding a twocomponent mass distribution whose potential is

$$
\begin{aligned}
\Phi_{\mathrm{h}}(R, z)= & \frac{v_{0}^{2}}{2} \ln \left(\tilde{R}_{\mathrm{h}}^{2}+\tilde{R}^{2}+\frac{\tilde{z}^{2}}{q_{\Phi}^{2}}\right) \\
& +\frac{G M_{0}}{r_{0}} \ln \left(\frac{r}{r_{0}+r}\right) .
\end{aligned}
$$

The first component represents a Binney (1981) logarithmic dark matter halo with asymptotic velocity $v_{0}$ and $\tilde{R}_{\mathrm{h}} \equiv R_{\mathrm{h}} / R_{\mathrm{d}}$; the corresponding potential belongs to the family of homeoidal potentials considered in Sect. 3.1.1. The second component, needed to reproduce the steep rising of the rotation curve in the innermost galaxy regions (see Fig. 1), is a Jaffe (1983) spherically symmetric density distribution of total mass $M_{0}$ and scalelength $r_{0}$.

The adopted values for the disk scale-length and central surface brightness $\left(R_{\mathrm{d}}=4.40 \mathrm{kpc}, \mu_{0 \mathrm{~B}}=21.4 \mathrm{mag} \mathrm{arcsec}^{-2}\right)$ were taken from Shaw \& Gilmore (1989). We find that the observed rotation curve of NGC 891 is well reproduced for $v_{0}=138 \mathrm{~km} \mathrm{~s}^{-1}, R_{\mathrm{h}}=R_{\mathrm{d}}, q_{\Phi}=0.71, M_{0}=2.40 \times 10^{10} M_{\odot}$, $r_{0}=1.15 \mathrm{kpc}$, and $M_{\mathrm{d}}=7.7 \times 10^{10} M_{\odot}$. The resulting disk B-band mass-to-light ratio is $\simeq 3.5$ (in solar units).

\subsection{The gas distribution and its rotational velocity field}

Having fixed the galaxy potential well, we now turn to the choice of the gas density distribution. Following the arguments presented in Sect. 3, we adopt the trial function

$\rho(R, z)=\frac{\rho_{0} \tilde{R}_{m}^{\beta}}{\tilde{R}_{0}^{\alpha}} \frac{\left(\tilde{R}_{0}+\tilde{R}\right)^{\alpha}}{\left(\tilde{R}_{m}^{2}+m^{2}\right)^{\beta / 2}} \mathrm{e}^{-\tilde{z} / h_{\mathrm{g}}}$,

where $m^{2} \equiv \tilde{R}^{2}+\tilde{z}^{2} / q_{\mathrm{g}}^{2}, \tilde{R}_{m} \equiv R_{m} / R_{\mathrm{d}}, \tilde{R}_{0} \equiv R_{0} / R_{\mathrm{d}}, \rho_{0}$ is the central gas, and $\beta>\alpha+2$ so that the total gas mass converges. Since the logarithmic halo belongs to the family of homeoidal potentials, $\rho$ is a factorized distribution similar to that of Eq. (12) with an homeoidally stratified component (flattening $\left.q_{\mathrm{g}}<q_{\Phi}\right)$, a cylindrical radially increasing factor $(\alpha>0)$ 

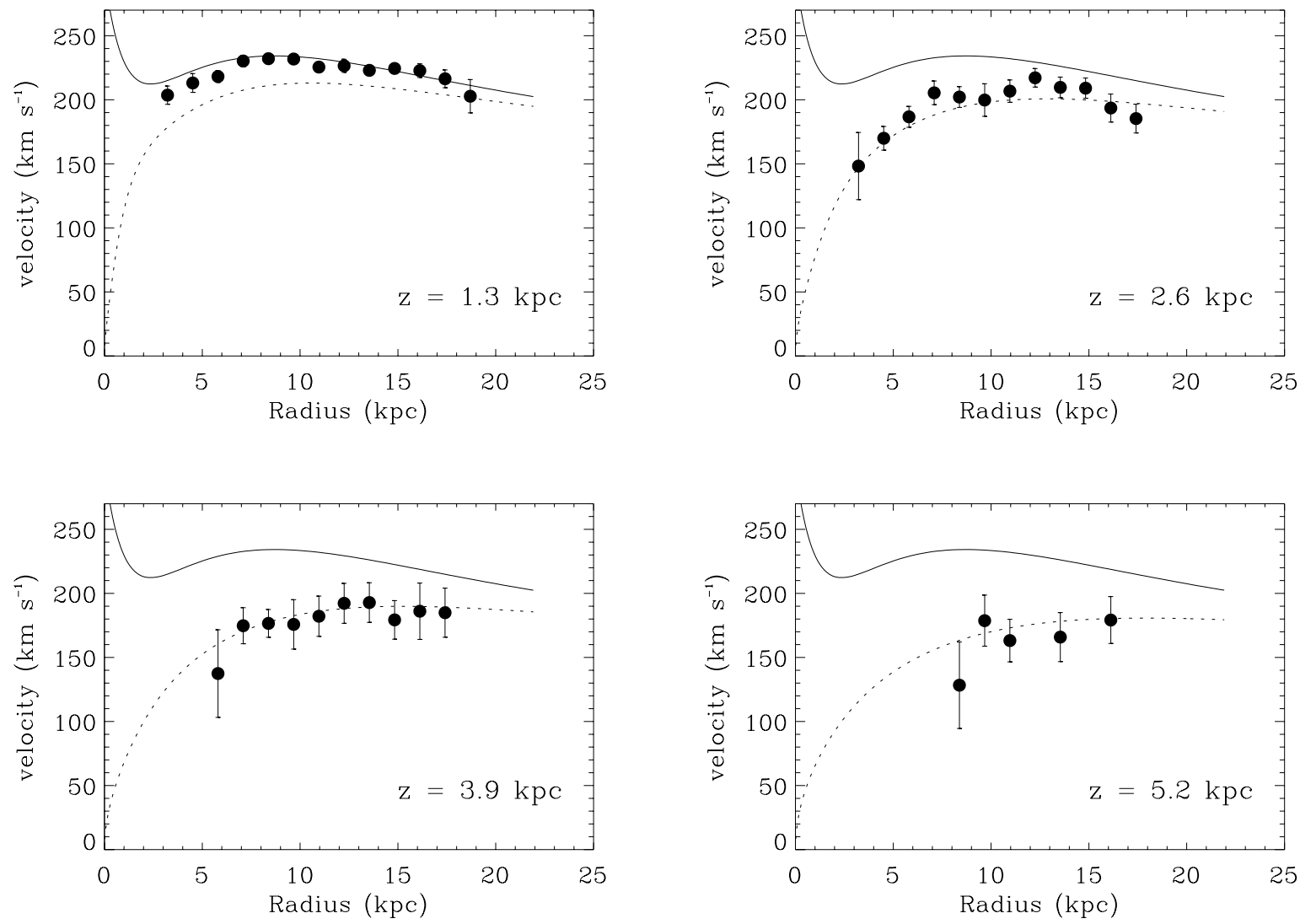

Fig. 2. Extra-planar H I rotation curves for NGC 891 (dots with error bars) at various heights over the galactic plane (from Fraternali et al. 2005). The solid curve is the rotation curve in the galactic plane $(z=0)$ as obtained from the model (see also Fig. 1). The dotted lines are the predicted baroclinic extra-planar rotation curves of the Reference Model.

and an isothermal distribution stratified on the gravity field of a razor-thin uniform disk.

We now solve Eqs. (1) for the present model. In particular, Eq. (2) is integrated numerically with a finite-difference scheme (Appendix C), obtaining the gas pressure, velocity and temperature values on the numerical grid.

The first result is that a large number of exploratory models (but not all of them), in which the value of the parameters in Eq. (20) is arbitrarily chosen, produce physically acceptable rotational velocities. This confirms the validity of the preparatory analysis in Sect. 3. For example, models without the radially increasing factor (obtained by setting $\alpha=0$ in Eq. (20)), invariably have $v_{\varphi}^{2}<0$ in the central regions, while models less flat than the halo potential $\left(q_{\mathrm{g}}>q_{\Phi}\right)$ turn out to be nonphysical in the far field. We conclude that the "core" properties of the physically acceptable models are the gas radial depression in the central regions and a gas distribution flatter than the halo potential at large distances. The remaining parameters play only a minor role.

We now present and discuss a specific, physically acceptable model, which we refer to as the "Reference Model" (RM). The free parameters of the RM have been fixed to reproduce the observed H I rotation curve of NGC 891 at $z=2.6 \mathrm{kpc}$ (Fraternali et al. 2005) and the resulting rotation curve is shown in Fig. 2 (upper right panel, dotted line). We have obtained an acceptable curve by adopting $\alpha=1, \beta=3.5, \tilde{R}_{0}=1$, $\tilde{R}_{m}=2.25, h_{\mathrm{g}}=1.5$ and $q_{\mathrm{g}}=0.1$. Note that, due to the assumption of a negligible self-gravity for the gas, from Eqs. (2) and (3) it follows that $v_{\varphi}$ and the temperature are independent of the specific choice of $\rho_{0}$. Other arguments will be used to fix the value of the latter (see Sect. 4.3).

In Fig. 3 we show the radial profiles of the RM gas density distribution at 5 different heights above the galactic plane (Fig. 3a) and the meridional sections of its isorotational (Fig. 3b) and isothermal (Fig. 3c) surfaces. From Fig. 3b we see that the rotation velocity decreases with $z$ at a fixed $R$, while Fig. $3 \mathrm{c}$ shows that the gas is warm, with temperatures ranging from $\sim 10^{4}$ to $\sim 10^{6} \mathrm{~K}$. The hotter gas is near the $R=0$ axis, in correspondence with the density decrease near the galaxy center, while the lowest gas temperatures are attained near the galactic plane.

In Fig. 2 we show the observed H I rotation curves at four different heights above the galactic plane $(z=1.3,2.6,3.9$, and $5.2 \mathrm{kpc}$, dots), and the rotation curves from the RM (dotted lines), corresponding to horizontal sections of Fig. 3b. The RM gas parameters have been tuned to obtain a good fit of the observed rotation curve at $z=2.6 \mathrm{kpc}$. This choice of parameters produces good fits for the curves at higher $z$ too. It is remarkable that the gas density distribution in Eq. (20), mainly built on theoretical arguments, should lead to a predicted rotational velocity decrease for the extra-planar gas which is so close to the observations. The discrepancy between data and model predictions at $z=1.3 \mathrm{kpc}$ could be real (see Sect. 4.3), but it is possible that the observed data are affected by the limited 

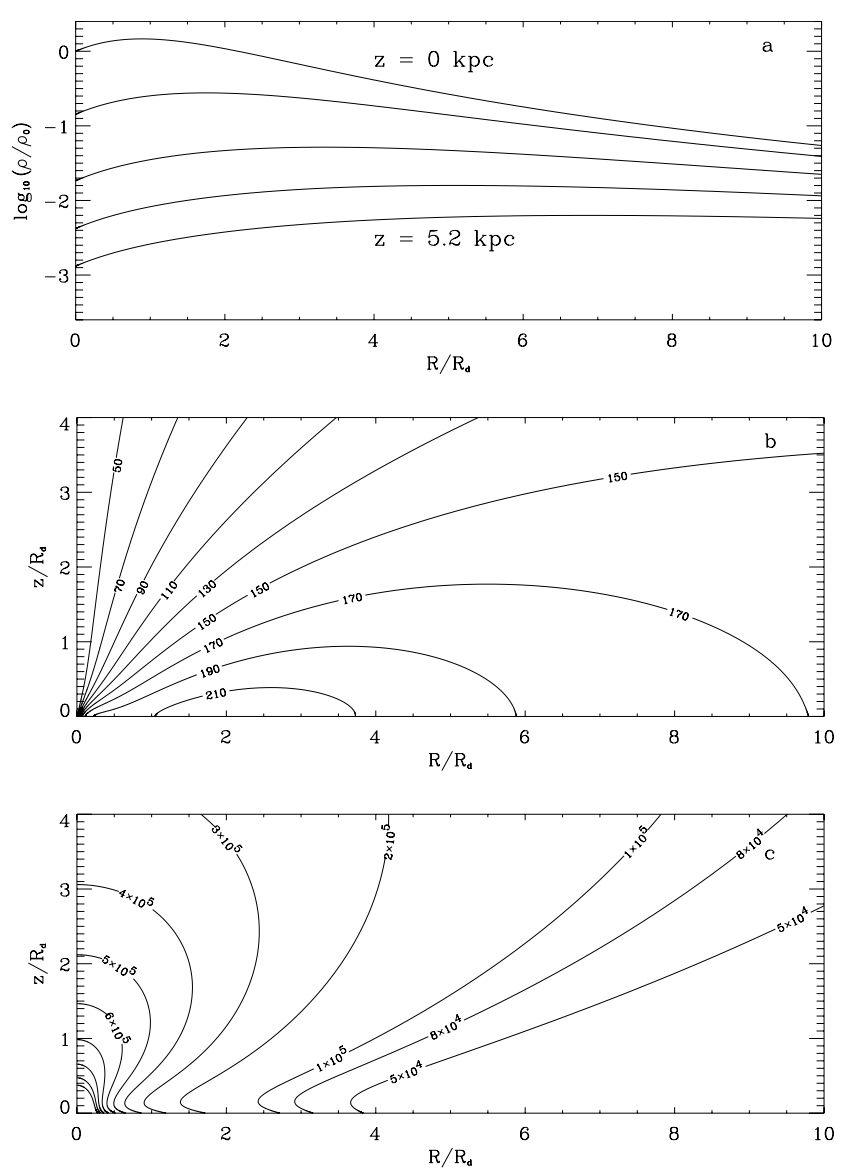

Fig. 3. Panel a): Radial profile of the density distribution for the Reference Model (RM) at $z=0,1.3,2.6,3.9$ and $5.2 \mathrm{kpc}$ (same heights as in Fig. 2). Panels b), c): RM isorotation curves $v_{\varphi}=$ const. (in $\mathrm{km} \mathrm{s}^{-1}$ ) and isothermal contours (in $\mathrm{K}$ ) in the meridional plane.

angular resolution of the observations $\left(H P B W=28^{\prime \prime} \approx\right.$ $1.3 \mathrm{kpc}$ ). Details on the derivation of the rotation curves from the observations will be presented in a forthcoming paper (Fraternali, in preparation).

How do the RM properties depend on the specific choice of the gas density distribution? Among the parameters, the largest effect is produced by the oblateness $q_{\mathrm{g}}$ and the vertical scaleheight $h_{\mathrm{g}}$. Increasing $q_{\mathrm{g}}$ has a major effect: isorotation curves would be heavily modified for $z \gtrsim R_{\mathrm{d}} / 2$, becoming almost vertical (cylindrical rotation) when $q_{\mathrm{g}} \simeq q_{\Phi}$, which is the limiting flattening according to condition (14). We note that a similar behaviour is also shown by the analytical model presented in Appendix A, even though this is based on a substantially simpler gas and galaxy model. A decrease of $h_{\mathrm{g}}$ has a strong effect for $z \gtrsim R_{\mathrm{d}}$, producing a cylindrical rotation pattern extending out to $R \simeq 5 R_{\mathrm{d}}$.

Variations of $\alpha, \beta$ and $\tilde{R}_{0}$ produce minor effects, at least in the regions covered by observations $\left(R \lesssim 5 R_{\mathrm{d}}\right.$ and $\left.z \lesssim 1.3 R_{\mathrm{d}}\right)$. In particular, $v_{\varphi}$ increases for increasing $\alpha$ and decreasing $\tilde{R}_{0}$, but the same overall structure of the RM is kept, and the differences are hardly detectable for $z \gtrsim 1.3 R_{\mathrm{d}}$. An increase of $\beta$ has an effect very similar to increasing $\alpha$, and again there is a corresponding behaviour in the analytical toy-model of Appendix A. Finally, an increase of $q_{\Phi}$ (i.e., the adoption of a rounder dark matter halo) at fixed $q_{\mathrm{g}}$ has only a very marginal effect on $v_{\varphi}$. We conclude that baroclinic solutions are sensitive to the adopted gas distributions, but only marginally to the halo flattening. It seems, therefore, that according to the fluid stationary interpretation presented here the kinematics of the extra-planar gas cannot be used as a sensitive diagnostic tool to investigate the flattening of dark matter haloes.

\subsection{Astrophysical interpretation}

The gas kinematics from the Reference Model is remarkably similar to that observed for the extra-planar $\mathrm{H}$; yet, the model gas temperature is much higher than that of HI (Fig. 3c). Does this mean that fluid solutions should be abandoned as unphysical?

The gas density distribution in Eq. (20) may be interpreted not only as that of a smooth, homogeneous fluid (for which pressure and temperature are the usual thermodynamical quantities), but also as the fluid description of the H I cloud distribution. In this paper we follow the first interpretation; however, a brief discussion of the second interpretation, in which Eqs. (1) are interpreted as the Jeans equations for a system with isotropic velocity dispersion, is given in Sect. 4.3.2 with further comments in Sect. 5.

\subsubsection{A homogeneous gas distribution}

Equilibrium configurations (baroclinic or not) of a homogeneous extra-planar gas are expected to be hotter than the $\mathrm{H}$ I because of the needed vertical pressure support against the galaxy gravitational field. Therefore, the observed cloudy extra-planar H I gas can not be described directly with fluid homogeneous models. We note, however, that baroclinic solutions as presented above are comparable to (or better than) ballistic models at reproducing the observed vertical gradients in the $\mathrm{H} \mathrm{I}$ and $\mathrm{H} \alpha$ rotational velocities. Furthermore, there is, in addition to cold $\mathrm{HI}$, hot gas in the halo of spiral galaxies as observed in some edge on systems, including NGC 891 (Bregman \& Pildis 1994; Strickland et al. 2004). It is therefore important to explore the possibility that the observed H I traces the kinematics of an underlying, homogeneous, hot gas.

The origin and the coexistence of hot and cold gas phases and their kinematical coupling are central issues in the discussion of possible realistic models for the extra-planar gas. An important question in this respect is how long it would take for the hot gas to cool if not resupplied with fresh energy, or to drag $\mathrm{H}$ I clouds (accreted or produced by galactic fountains) in regular motion around the galaxy axis. Clearly, hydrodynamical numerical simulations are needed to fully address this. Here, we limit ourselves to a first order analysis of the main astrophysical aspects and implications of the Reference Model presented above.

Information on the thermal evolution of the RM is obtained from its local cooling time

$t_{\text {cool }}(R, z) \equiv \frac{E}{\dot{E}} \propto \frac{1}{\rho_{0}}$, 
where $E=3 \rho k T / 2 \mu m_{\mathrm{p}}$ and $\dot{E}=n_{\mathrm{e}} n_{\mathrm{p}} \Lambda_{0}(T)$ are the gas internal energy and cooling rate per unit volume; $k, \mu, m_{\mathrm{p}}, n_{\mathrm{e}}$ and $n_{\mathrm{p}}$ are the Boltzmann constant, the gas mean molecular weight, the proton mass, and the number density of electrons and protons, respectively. We also adopt the cooling function

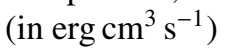

$\Lambda_{0}(T) \simeq\left\{\begin{array}{l}5.36 \times 10^{-27} T, \\ 2.18 \times 10^{-18} T^{-0.6826}+2.71 \times 10^{-47} T^{2.976},\end{array}\right.$

where the first equation holds for $10^{4} \leq T \leq 1.3 \times 10^{5} \mathrm{~K}$, and the second for $1.3 \times 10^{5} \leq T \leq 10^{8} \mathrm{~K}$ (Mathews \& Bregman 1978, see also Ciotti et al. 1991). For simplicity we assume $n_{\mathrm{e}}=n_{\mathrm{p}}(\mu=1 / 2)$ and so

$\dot{E}=\frac{\rho^{2}}{m_{\mathrm{p}}^{2}} \Lambda_{0}(T)$.

Note that, at variance with $v_{\varphi}$ and $T, \dot{E}$ and $t_{\text {cool }}$ do depend on the value of the gas density normalization constant $\rho_{0}$ in Eq. (20), and so we are now forced to fix its value. A simple argument can be based on the requirement of stationarity: the gas distribution must radiate per unit time a total amount of energy of the same order of magnitude as that provided by supernova explosions and stellar winds in the stellar disks of NGC 891, estimated $\approx 2.9 \times 10^{42} \mathrm{erg} \mathrm{s}^{-1}$ (Bregman \& Houck 1997, and references therein). The total (bolometric) luminosity of the gas distribution is

$L_{\mathrm{tot}}=4 \pi \int_{0}^{\infty} \int_{0}^{\infty} \dot{E} R \mathrm{~d} R \mathrm{~d} z \propto \rho_{0}^{2}$,

and from numerical integration we find that $\rho_{0} \approx 2.4 \times$ $10^{-26} \mathrm{~g} \mathrm{~cm}^{-3}$, corresponding to a total gas mass $M_{\mathrm{gas}} \approx 1.6 \times$ $10^{9} M_{\odot}$ (it should be noted that use of $n_{\mathrm{e}}=1.2 n_{\mathrm{p}}$ to include helium ionization would lead to a density normalization and to a total mass $\approx 10 \%$ smaller than the values obtained here). The hypothesis of a negligible gas self-gravity on a galactic scale is thus satisfied, the resulting gas mass being only a few percent of the total mass of the galaxy. Curiously, the mass of the extra-planar gas distributed according to $(20)\left(\approx 5.5 \times 10^{8} M_{\odot}\right.$ for $z \gtrsim 1.4 \mathrm{kpc})$ is found to be of the same order of magnitude as the mass of the observed extra-planar H I $\left(\approx 6 \times 10^{8} M_{\odot}\right.$, Swaters et al. 1997), while the luminosity of the RM extraplanar gas is very low, only $\approx 5 \%$ of the estimated energy injection rate. From this point of view, the bulk of the supernova and stellar wind heating is radiated by the gas near the galactic disk, in good quantitative agreement with the conclusions of Read \& Ponman (1995). This is confirmed by the surface brightness distribution $\Sigma$ of the RM, obtained projecting $\dot{E}(R, z)$ along the line-of-sight. When the galaxy is seen edge-on, the surface brightness is given by:

$\Sigma(R, z)=2 \int_{R}^{\infty} \frac{\dot{E} R^{\prime} \mathrm{d} R^{\prime}}{\sqrt{R^{\prime 2}-R^{2}}}$.

In Fig. 4a we show the RM bolometric surface brightness distribution. We have also computed the RM luminosity for the gas with $T \geq 5.5 \times 10^{5} \mathrm{~K}$ (that we identify with EUV-emitting gas), obtaining $L_{\mathrm{EUV}} \sim 10^{-2} L_{\mathrm{tot}}$, with the bulk of the emission
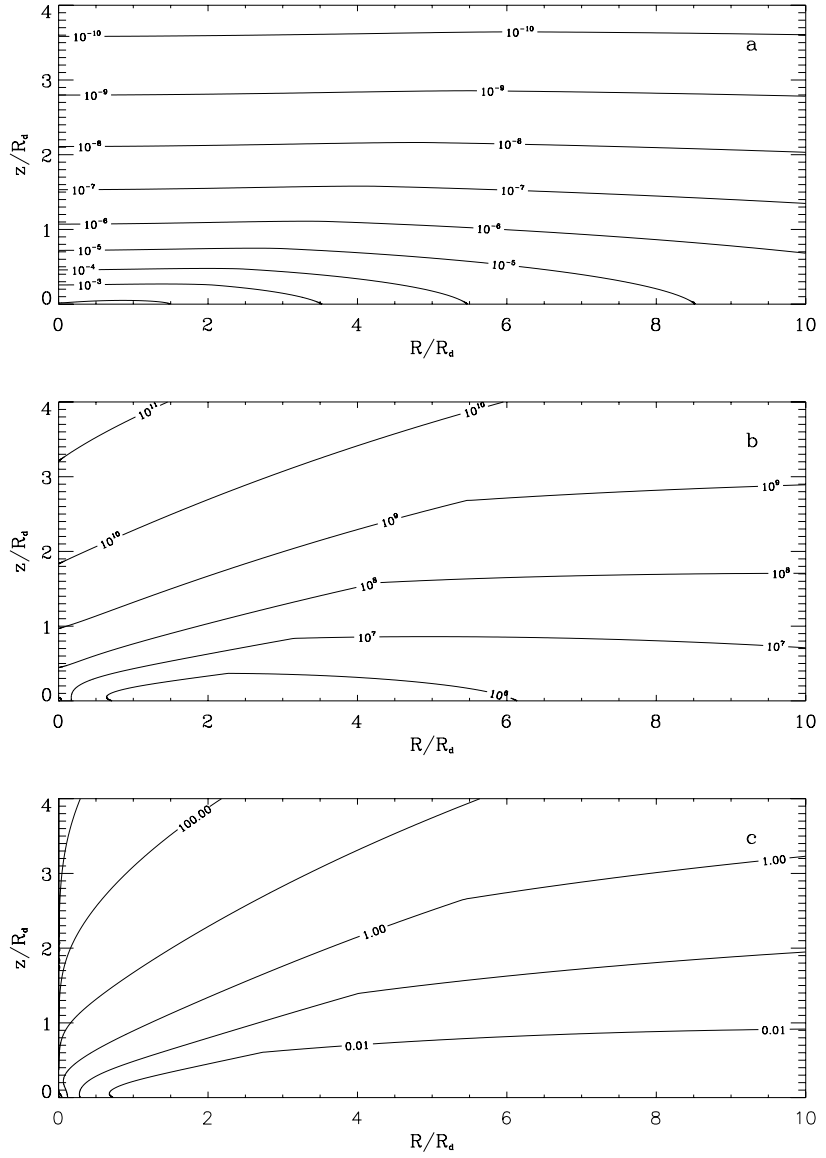

Fig. 4. Panel a): Bolometric surface brightness isophotes (in erg $\mathrm{s}^{-1}$ $\mathrm{cm}^{-2}$ ) for the RM. Panel b): meridional sections of equal cooling time (in yrs) surfaces for the RM with $\rho_{0}=2.4 \times 10^{-26} \mathrm{~g} \mathrm{~cm}^{-3}$. Panel c): contours of equal $\xi=t_{\text {cool }} / t_{\mathrm{dyn}}$ (Eq. (27)) in the meridional plane.

confined in a hot bubble along the galactic axis and matching the temperature distribution in Fig. 3c.

The conclusion that a major fraction of the galaxy heating is radiated near the disk is also confirmed by the fact that cooling times increase from the galactic plane toward regions of higher $z$ and lower gas density, with values in the range $10^{6} \lesssim t_{\text {cool }} \lesssim 10^{11}$ yrs (Fig. 4b). Obviously, the gas in the regions where $t_{\text {cool }}$ is short will not remain homogeneous and its natural fate will be to cool and form clouds and filaments. The energy input from the disk would be then used mainly to maintain a time-average, stationary multiphase structure.

Additional insight into this multiphase medium is provided by the dynamical time

$t_{\mathrm{dyn}}(R, z) \equiv \frac{2 \pi R}{v_{\varphi}(R, z)}$,

i.e., the orbital period of a gas element in circular orbit at $(R, z)$. We introduce here the dimensionless number

$\xi \equiv \frac{t_{\mathrm{cool}}}{t_{\mathrm{dyn}}} \propto \frac{1}{\rho_{0}}$.

From Fig. $4 \mathrm{c}$ it appears that $\xi \gtrsim 1$ for $z \gtrsim 1 \div 2 R_{\mathrm{d}}$ and near the galaxy axis. It is expected that where $\xi<1$ the gas will cool locally. Note that the regions where $\xi<1$ match very closely those in which $t_{\text {cool }}<1$ Gyr. 
In order to address the issue of the interaction of $\mathrm{HI}$ clouds with the hot and homogeneous baroclinic gas distribution of the $\mathrm{RM}$, we have computed the cloud drag time $t_{\mathrm{drag}}$, i.e. the time required for an $\mathrm{H}$ I cloud to corotate with the homogeneous gas halo described by Eq. (20). This quantity is relevant whatever the origin of the H I clouds: thermal instabilities of the hot gas, galactic fountains or cosmological accretion. Following Klein et al. (1994), we define

$t_{\mathrm{drag}}(R, z) \equiv \frac{8}{3 C_{\mathrm{D}}} \frac{r_{\mathrm{c}}}{v_{\text {rel }}} \chi \propto \frac{1}{\rho_{0}}$,

where $C_{\mathrm{D}} \simeq 1$ is a numerical coefficient, $r_{\mathrm{c}}$ is the radius of a typical H I cloud, $v_{\text {rel }}$ is the modulus of the relative velocity between the cloud and the homogeneous extra-planar component, and $\chi \equiv \rho_{\mathrm{c}} / \rho$ is the ratio between the cloud and the medium densities.

We estimate $t_{\text {drag }}$ assuming pressure equilibrium between cold and hot gas components, from which $\chi=T / T_{\mathrm{c}} \approx 3000$. A fiducial value of the relative velocity is obtained by assuming $v_{\text {rel }}=v_{\varphi} \approx 2 \times 10^{7} \mathrm{~cm} \mathrm{~s}^{-1}$, while the cloud radius is estimated as $4 \pi r_{\mathrm{c}}^{3} \rho_{\mathrm{c}} / 3=10^{5} M_{\odot} \tilde{M}_{5}$, where $\tilde{M}_{5}$ is the cloud mass in units of $10^{5} M_{\odot}$. We thus obtain

$$
\begin{aligned}
t_{\text {drag }} & \simeq \frac{8}{3 v_{\varphi}}\left(\frac{3 \times 10^{5} M_{\odot} \tilde{M}_{5}}{4 \pi n_{\mathrm{p}} m_{\mathrm{p}}}\right)^{1 / 3}\left(\frac{T}{T_{\mathrm{c}}}\right)^{2 / 3} \\
& \approx 2.7 \times 10^{8}\left(\frac{\tilde{M}_{5}}{n_{\mathrm{p}}}\right)^{1 / 3} \mathrm{yrs},
\end{aligned}
$$

where $\rho=n_{\mathrm{p}} m_{\mathrm{p}}$. From this simple formula it is apparent that massive clouds at high $z$ above the galactic plane cannot be dragged by the hot and tenuous RM distribution (see Fig. 3a for characteristic values of $n_{\mathrm{p}}$ ), while smaller clouds (e.g. $\left.\tilde{M}_{5} \sim 10^{-3}\right)$ near the disk $(z \approx 1 \div 2 \mathrm{kpc})$ have drag times significantly shorter than rotational times. It cannot be excluded, therefore, that extraplanar H I clouds (or, even better, H I filaments, which have larger cross-sections) could be the tracers of the underlying fluid. Of course, if H I clouds originated from cosmological accretion, then the proper characteristic time to be compared with $t_{\text {drag }}$ is the free-fall time. However, $t_{\mathrm{dyn}}$ is of the same order of magnitude and the above arguments should still be valid. In any case, only hydrodynamical simulations will possibly clarify the importance of drag and related issues, such as the angular momentum redistribution between the homogeneous gas and the H I clouds. Thus, our analysis seems to leave open the possibility advocated by Collins et al. of the presence of a non-gravitational forces acting on the extraplanar H I gas.

\subsubsection{A "gas" of $\mathrm{HI}$ clouds}

As pointed out at the beginning of Sect. 4, from a formal point of view, Eqs. (1) are identical to the fluid stationary Jeans equations for an axisymmetric system with a globally isotropic velocity dispersion tensor. Thus, for such a system we would also obtain rotational velocities decreasing with increasing $z$. The pressure field, however, must be interpreted as $P=\rho \sigma^{2}$, where $\sigma$ is the cloud (1-dimensional) velocity dispersion, so that $\sigma=\sqrt{k T / \mu m_{\mathrm{p}}} \approx 41 \sqrt{\tilde{T}_{5}} \mathrm{~km} \mathrm{~s}^{-1}$, where $\tilde{T}_{5} \equiv T / 10^{5} \mathrm{~K}$.
From Fig. 3c it appears that near the disk the clouds velocity dispersion would be of the order of $30 \mathrm{~km} \mathrm{~s}^{-1}$ or even less, while higher values of $\sigma$ are expected near the galaxy center. Such values might not be unreasonable. However, a further comparison of the hot and homogeneous gas solution with the present one is not straightforward: while the RM gas distribution in Eq. (20) was adopted to describe a hot and homogeneous gas distribution (and thus not very well constrained by observations), a proper Jeans-based analysis would require the choice of a cloud distribution similar to that observed. In principle this should be simple to do, but we do not explore this any further here. Even if the temperature and related problems addressed in Sect. 4.3.1 were solved, in the Jeans-based interpretation the question of how the cloud velocity dispersion tensor is rendered isotropic would become the new central question.

\section{Discussion and conclusions}

We have presented a family of fluid stationary models for the extra-planar gas in spiral galaxies. As an application, we have built a model for the extra-planar gas of the spiral galaxy NGC 891. The main results of our analysis are:

(i) Physically acceptable baroclinic solutions exist for gas density distributions (isodensity surfaces) more flattened than the isopotential surfaces. For regions near the disk, the condition is that the gas density distribution is centrally depressed.

(ii) Application of our method to the case of the edge-on galaxy NGC 891 has shown that with baroclinic solutions it is possible to reproduce the observed vertical decrease of the gas rotational velocity.

(iii) In homogeneous fluid stationary solutions the gas temperature is in the range $10^{4} \lesssim T \lesssim 10^{6} \mathrm{~K}$, well above the $\mathrm{HI}$ temperature and the cooling times are short, of the order of the orbital times.

(iv) In the hot, homogeneous gas configurations with negative velocity gradients of the present models, the drag on small H I clouds $\left(\sim 10^{2} M_{\odot}\right)$ near the disk is important and may account for the observed vertical velocity decrease. It is not certain, however, that this applies to the case of NGC 891 where the H I structures may be more massive.

(v) Instead of a hot homogeneous medium, the baroclinic solution can also provide a good fluid description of a "gas" of clouds (according to the stationary Jeans equations) with a globally isotropic velocity dispersion tensor. All the above considerations about the decrease of rotational velocity with increasing $z$ would still hold, while the temperature field would have to be interpreted as the velocity dispersion field.

Ballistic models have been tried for the extra-planar gas. It seems, however, that in order to reproduce the observed rotational velocity gradients, non-gravitational effects, such as gas and magnetic pressure, also may be necessary (Collins et al. 2002; Fraternali \& Binney 2005). Our analysis seems to suggest that such non gravitational effects could be due (at least for low mass clouds) to ram pressure. $t_{\mathrm{drag}}$ depends on the relative velocity between the H I clouds and the hot baroclinic gas, 
hence the effect of the drag would be to regularize the motion of the clouds to the velocity field of the embedding medium. For example, if the H I clouds are ejected from the disk by galactic fountains, on average they will be initially faster with respect to the baroclinic gas and will be slowed down to its velocity while rising. The drag could also be effective in regularizing the dynamics of accreted clouds: from this point of view, regular H I dynamics (in particular, the same sense of rotation of the galactic disk) would not necessarily be an indication of an internal origin of the extra-planar gas.

The problem of stability of the models presented here is a difficult one and remains open. Numerical hydrodynamical simulations would be very useful to address this. In the approach adopted in this paper, where the density distribution for the extraplanar gas is assigned, pressure, temperature and rotation cannot be arbitrarily prescribed but are determined by the galaxy gravitational potential. The stability of such configurations is not guaranteed. Additional effects needing quantitative estimates are the drag of clouds (both of internal and external origin), their cooling and evaporation times, and the overall energy budget, to follow up on the pioneering work of Cox \& Smith (1974) and McKee \& Ostriker (1977). Equally important would be the numerical study of baroclinic solutions in the context of Jeans equations. In this case, isotropy of the velocity dispersion tensor could be provided by cloud-cloud collisions, a physical ingredient usually neglected in numerical simulations (however, see Waxman 1978). One could speculate that the problems encountered by purely ballistic models are due to the absence of a collision term in the simulations, as already suggested by Collins et al. (2002).

Fluid-homogeneous and Jeans-based baroclinic models are not mutually exclusive. They could be integrated and used together for a better understanding of the dynamics of the extraplanar gas.

Acknowledgements. We thank the anonymous referee for insightful comments. We are grateful to Giuseppe Bertin, James Binney, Jeremiah Ostriker, and Hugo van Woerden for enlightening discussions. We also thank Fabrizio Brighenti and Annibale D'Ercole for useful comments. M.B. was partially supported by ASI contract IR/063/02 and by INAF-Bologna Astronomical Observatory. L.C. and R.S. were supported by the grant CoFin2004 (MIUR).

\section{References}

Barnabè, M., Ciotti, L., Fraternali, F., \& Sancisi, R. 2005, in Extraplanar Gas Conference, ed. R. Braun, ASP Conf. Ser. 331, 231

Benjamin, R. A. 2002, in Seeing Through the Dust: the Detection of H I and the Exploration of the ISM in Galaxies, ed. A. R. Taylor, T. L. Landecker, \& A. G. Willis (San Francisco: ASP), ASP Conf. Ser., 276, 201
Binney, J. 1981, MNRAS, 196, 455

Binney, J. 2005, in Extra-planar Gas Conference, ed. R. Braun, ASP Conf. Ser., 331, 131

Binney, J., \& Tremaine, S. 1987, Galactic Dynamics (Princeton: Princeton University Press)

Boomsma, R., Oosterloo, T., Fraternali, F., van der Hulst, T., \& Sancisi, R. 2005, in Extra-planar Gas Conference, ed. R. Braun, ASP Conf. Ser., 331, 247

Boulares, A., \& Cox, D. P. 1990, ApJ, 365, 544

Bregman, J. N. 1980, ApJ, 236, 577

Bregman, J. N., \& Pildis, R. A. 1994, ApJ, 420, 570

Bregman, J. N., \& Houck, J. C. 1997, ApJ, 485, 159

Cayatte, V., Kotanyi, C., Balkowski, C., \& van Gorkom, J. H. 1994, AJ, 107, 1003

Ciotti, L., \& Bertin, G. 2005, A\&A, 437, 419

Ciotti, L., D'Ercole, A., Pellegrini, S., \& Renzini, A. 1991, ApJ, 376, 380

Collins, J. A., Benjamin, R. A., \& Rand, R. J. 2002, ApJ, 578, 98

Cox, D. P., \& Smith, B. W. 1974, ApJ, 189, L105

Evans, N. W. 1994, MNRAS, 267, 333

Fraternali, F., \& Binney, J. 2005, MNRAS, submitted

Fraternali, F., Oosterloo, T., Boomsma, R., Swaters, R., \& Sancisi, R. 2004, in Recycling Intergalactic and Interstellar Matter, ed. P.-A. Duc, J. Braine, \& E. Brinks (Sidney: IAU), IAU Symp., 217, 136 Fraternali, F., Oosterloo, T., \& Sancisi, R. 2004, A\&A, 424, 485

Fraternali, F., Oosterloo, T., Sancisi, R., \& Swaters, R. 2005, in Extraplanar Gas Conference, ed. R. Braun, ASP Conf. Ser., 331, 239

Hunter, C. 1977, AJ, 82, 271

Jaffe, W. 1983, MNRAS, 202, 995

Klein, R. I., McKee, C. F., \& Colella, P. 1994, ApJ, 420, 213

Lanzoni, B., \& Ciotti, L. 2003, A\&A, 404, 819

Lebovitz, N. R. 1967, ARA\&A, 5, 465

Mathews, W. G., \& Bregman, J. N. 1978, AJ, 224, 308

Matthews, L. D., \& Wood, K. 2003, AJ, 593, 721

McKee, C. F., \& Ostriker, J. P. 1977, ApJ, 218, 148

Puche, D., Westpfahl, D., Brinks, E., \& Roy, J.-R. 1992, AJ, 103, 1841

Rand, R. J. 1997, ApJ, 474, 129

Rand, R. J. 2000, ApJ, 537, L13

Read, A. M., \& Ponman, T. J. 1995, MNRAS, 276, 1327

Rossa, J., Dettmar, R.-J., Walterbos, R. A. M., \& Norman, C. A. 2004, AJ, 128, 674

Rosseland, S. 1926, ApJ, 63, 342

Shapiro, P. R., \& Field, G. B. 1976, ApJ, 205, 762

Shaw M. A., \& Gilmore G. 1989, MNRAS, 237, 903

Strickland, D. K., Heckman, T. M., Colbert, E. J. M., Hoopes, C. G., \& Weaver, K. A. 2004, ApJS, 151, 193

Swaters, R. A., Sancisi, R., \& van der Hulst, J. M. 1997, ApJ, 491, 140

Tassoul, J.-L. 1980, Theory of Rotating Stars (Princeton: Princeton University Press)

Wang, Q. D., Immler, S., Walterbos, R., Lauroesch, J. T., \& Breitschwerdt, D. 2001, ApJ, 555, L99

Waxman, A. M. 1978, ApJ, 222, 61 


\section{Online Material}




\section{Appendix A: A simple analytical model}

We present here a simple and fully analytical model of a baroclinic gas distribution characterized by the vertical decline of the rotational velocity. The dimensionless gas density distribution

$\tilde{\rho}=\frac{1-\eta_{\mathrm{g}} \lambda}{\tilde{r}^{\lambda}}+\frac{\eta_{\mathrm{g}} \lambda \tilde{R}^{2}}{\tilde{r}^{2+\lambda}}, \quad(0<\lambda<3)$,

is obtained from a homeoidal expansion of the oblate powerlaw distribution $\rho_{0} / m^{\lambda}$, where $\tilde{\rho} \equiv \rho / \rho_{0}, r=\sqrt{R^{2}+z^{2}}, m^{2}=$ $\tilde{R}^{2}+\tilde{z}^{2} /\left(1-\eta_{\mathrm{g}}\right)^{2}, \tilde{R} \equiv R / R_{\mathrm{g}}, \tilde{z} \equiv z / R_{\mathrm{g}}$, and $0 \leq \eta_{\mathrm{g}} \leq 1 / \lambda$ is the flattening of the distribution (Ciotti \& Bertin 2005).

The galaxy (stars plus dark matter) density distribution is also described by the homeoidal expansion of $\rho_{0 \mathrm{~h}} / \mathrm{m}^{\gamma}$, (where $0<\gamma<3, \rho_{0 \mathrm{~h}}$ is the normalization density, $m$ is defined as above but with $\eta_{\mathrm{h}}$ replacing $\eta_{\mathrm{g}}$, and $\left.0 \leq \eta_{\mathrm{h}} \leq 1 / \gamma\right)$. Without loss of generality the galaxy scale-length is $R_{\mathrm{g}}$, and the gravitational potential for $\gamma \neq 2$ (in units of $4 \pi G R_{\mathrm{g}}^{2} \rho_{0 \mathrm{~h}}$ ) is given by

$\tilde{\Phi}=-\frac{5-\gamma-\eta_{\mathrm{h}}(4-\gamma)(\gamma-1)}{(5-\gamma)(3-\gamma)(\gamma-2) \tilde{r}^{\gamma-2}}-\frac{\eta_{\mathrm{h}} \tilde{R}^{2}}{(5-\gamma) \tilde{r}^{\gamma}}$

(Ciotti \& Bertin, Eq. (27)). The pressure (normalized to $\left.4 \pi G R_{\mathrm{g}}^{2} \rho_{0 \mathrm{~h}} \rho_{0}\right)$ and the square of the gas rotational velocity field (normalized to $4 \pi G R_{\mathrm{g}}^{2} \rho_{0 \mathrm{~h}}$ ) are obtained from Eqs. (2)-(3). For simplicity we report here the solutions up to the linear terms in the flattenings, even though the derivation of the full solutions (i.e., including also the terms in $\eta_{\mathrm{h}} \eta_{\mathrm{g}}$ ) presents no difficulty. Accordingly,

$$
\begin{aligned}
\tilde{P}= & \frac{\tilde{r}^{2-\lambda-\gamma}}{(5-\gamma)(3-\gamma)}\left[\frac{(5-\gamma)\left(1-\eta_{\mathrm{g}} \lambda\right)-(4-\gamma)(\gamma-1) \eta_{\mathrm{h}}}{\gamma+\lambda-2}\right. \\
& \left.+\frac{(3-\gamma) \gamma \eta_{\mathrm{h}}+(5-\gamma) \eta_{\mathrm{g}} \lambda}{\gamma+\lambda} \frac{\tilde{R}^{2}}{\tilde{r}^{2}}\right], \\
\tilde{\rho} \tilde{v}_{\varphi}^{2}= & \frac{2 \lambda \tilde{R}^{2}}{(\gamma+\lambda) \tilde{r}^{\gamma+\lambda}}\left(\frac{\eta_{\mathrm{g}}}{3-\gamma}-\frac{\eta_{\mathrm{h}}}{5-\gamma}\right) .
\end{aligned}
$$

For $\eta_{\mathrm{g}}<\eta_{\mathrm{h}}(3-\gamma) /(5-\gamma)$ the (linearized) solution is unphysical. The isorotation curves for a physically acceptable case (obtained from the full solution) are shown in Fig. A.1, where it is apparent how, even in this extremely simplified model, the rotational velocity decreases with increasing $z$.

\section{Appendix B: How to construct $\rho_{\mathrm{e}}\left(\Phi_{\text {eff }}\right)$}

A first approach to the construction of the distribution $\rho_{\mathrm{e}}\left(\Phi_{\mathrm{eff}}\right)$ considered in Sect. 3.1 is the assumption that $P=P(\rho)$, as for example $P \propto \rho^{\gamma}$. In this case, from Eq. (9)

$$
\rho_{\mathrm{e}}\left(\Phi_{\mathrm{eff}}\right)=\rho_{0} \times\left\{\begin{array}{l}
\exp \left[-\frac{\mu m_{\mathrm{p}}}{k T_{0}}\left(\Phi_{\mathrm{eff}}-\Phi_{\mathrm{eff}, 0}\right)\right], \\
{\left[1+\frac{\gamma-1}{\gamma} \frac{\mu m_{\mathrm{p}}}{k T_{0}}\left(\Phi_{\mathrm{eff}, 0}-\Phi_{\mathrm{eff}}\right)\right]^{\frac{1}{\gamma-1}},}
\end{array}\right.
$$

where $\rho_{0}$ and $\Phi_{\text {eff }, 0}$ are taken at the same arbitrary but fixed point $\left(R_{0}, z_{0}\right)$, and the first expression holds for the isothermal

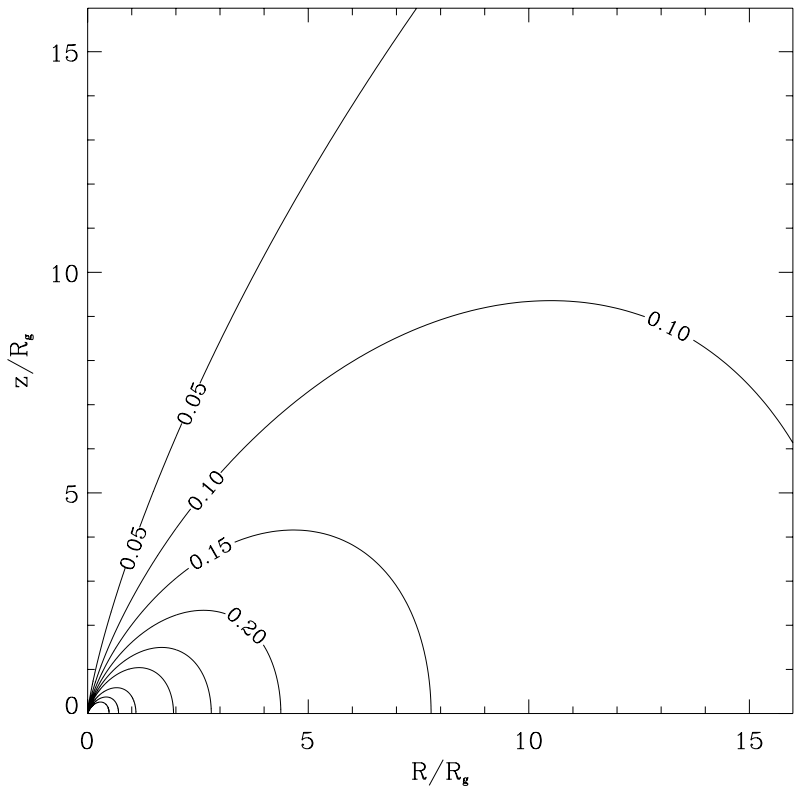

Fig. A.1. Meridional section of the isorotation surfaces $\tilde{v}_{\varphi}^{2}$ (labels along the curves) for $\gamma=2.5, \eta_{\mathrm{h}}=0.2, \lambda=2.3$ and $\eta_{\mathrm{g}}=0.3$.

case. In the second case $T / T_{0}=\left(\rho_{\mathrm{e}} / \rho_{0}\right)^{\gamma-1}$ and, at variance with the isothermal case, a truncation of $\rho_{\mathrm{e}}$ may appear. A different approach to the construction of $\rho_{\mathrm{e}}$ is however possible, where the specific density field is prescribed. This can be done by using the well known property that solutions of Eq. (9) necessarily are stratified on $\Phi_{\text {eff }}$, i.e. $\rho_{\mathrm{e}}=\rho_{\mathrm{e}}\left(\Phi_{\mathrm{eff}}\right)$ and $P=P\left(\Phi_{\text {eff }}\right)$. Thus, if one fixes $\Phi_{\text {eff }}$ and a prescribed function $\rho_{\mathrm{e}}\left(\Phi_{\text {eff }}\right)$, the pressure field is obtained by direct integration as

$P\left(\Phi_{\mathrm{eff}}\right)=P\left(\Phi_{\mathrm{eff}, 0}\right)-\int_{\Phi_{\mathrm{eff}, 0}}^{\Phi_{\mathrm{eff}}} \rho_{\mathrm{e}}(t) \mathrm{d} t$

Of course, this approach can be used only for density stratifications such that $P>0$ everywhere.

\section{Appendix C: The numerical code}

We describe here the scheme on which the (double precision) FORTRAN77 code adopted for the construction of models in Sect. 4 is based. From symmetry arguments we restrict the computation to the region $\left[R_{\min }, R_{\max }\right] \times\left[z_{\min }, z_{\max }\right]$ in the half-space $z \geq 0$, on which we define a bilogarithmic grid of $\left(n_{R}+1\right) \times\left(n_{z}+1\right)$ elements. In the radial direction the grid is

$R_{i}=R_{1} \times 10^{(i-1) \Delta R}$

where $R_{0}=0, R_{1}=R_{\min }$, and

$\Delta R \equiv \frac{\log R_{\max }-\log R_{\min }}{n_{R}-1}$,

so that $R_{\max }=R_{n_{R}}$; the $z$ coordinate is discretized in the same way.

The gas density and the gravitational field components at $\left(R_{i}, z_{j}\right)$ are $\rho_{j}^{i},{ }_{R} \Phi_{j}^{i} \equiv(\partial \Phi / \partial R)_{i j}$ and ${ }_{z} \Phi_{j}^{i} \equiv(\partial \Phi / \partial z)_{i j}$. For fixed 
M. Barnabè et al.: Hydrostatic models for the rotation of extra-planar gas, Online Material p 3

$R_{i}$ we integrate the first of Eqs. (1) with linear interpolation and boundary condition $P\left(R_{i}, z_{\max }\right)=0$ :

$P_{j}^{i}=\frac{1}{2} \sum_{k=j}^{n_{z}-1} \Delta z_{k} \times\left(\rho_{k z}^{i} \Phi_{k}^{i}+\rho_{k+1 z}^{i} \Phi_{k+1}^{i}\right)$,

where $P_{j}^{i} \equiv P\left(R_{i}, z_{j}\right)$ and $\Delta z_{k} \equiv z_{k+1}-z_{k}$. Accordingly, from Eq. (3):

$\rho_{j}^{i}\left(v_{\varphi}^{2}\right)_{j}^{i}=\left({ }_{R} P_{j}^{i}+{ }_{R} \Phi_{j}^{i} \rho_{j}^{i}\right) R_{i}$,

where ${ }_{R} P_{j}^{i} \equiv\left(P_{j}^{i+1}-P_{j}^{i}\right) / \Delta R_{i}$ and $\Delta R_{i} \equiv R_{i+1}-R_{i}$. The gas temperature $T$, the cooling rate $\dot{E}$ (Eq. (23)), the cooling time $t_{\text {cool }}$ (Eq. (21)) and the ratio $\xi$ between cooling time and dynamical time (Eq. (27)) are then obtained on the grid points.

The gas total luminosity is obtained as

$$
L_{\mathrm{tot}}=\sum_{i=0}^{n_{R}-1} \sum_{j=0}^{n_{z}-1} L_{j}^{i}
$$

where

$L_{j}^{i}=4 \pi \int_{R_{i}}^{R_{i+1}} \int_{z_{j}}^{z_{j+1}} R \dot{E}(R, z) \mathrm{d} R \mathrm{~d} z$,

and $\dot{E}(R, z)$ is evaluated on the numerical grid from Eq. (23). The integral in Eq. (C.6) is evaluated analitically in each region $\left[R_{i}, R_{i+1}\right] \times\left[z_{j}, z_{j+1}\right]$ by considering the bilinear expansion

$\dot{E}(R, z)=A_{0}+A_{1} R+A_{2} z+A_{3} R z$, where

$$
\begin{aligned}
A_{0} \equiv & \dot{E}_{j}^{i}-\frac{\dot{E}_{j}^{i+1}-\dot{E}_{j}^{i}}{\Delta R_{i}} R_{i}-\frac{\dot{E}_{j+1}^{i}-\dot{E}_{j}^{i}}{\Delta z_{j}} z_{j} \\
& +\frac{\dot{E}_{j+1}^{i+1}-\dot{E}_{j}^{i+1}+\dot{E}_{j}^{i}-\dot{E}_{j+1}^{i}}{\Delta R_{i} \Delta z_{j}} R_{i} z_{j}
\end{aligned}
$$

$A_{1} \equiv \frac{\dot{E}_{j}^{i+1}-\dot{E}_{j}^{i}}{\Delta R_{i}}-\frac{\dot{E}_{j+1}^{i+1}-\dot{E}_{j}^{i+1}+\dot{E}_{j}^{i}-\dot{E}_{j+1}^{i}}{\Delta R_{i} \Delta z_{j}} z_{j}$

$A_{2} \equiv \frac{\dot{E}_{j+1}^{i}-\dot{E}_{j}^{i}}{\Delta z_{j}}-\frac{\dot{E}_{j+1}^{i+1}-\dot{E}_{j}^{i+1}+\dot{E}_{j}^{i}-\dot{E}_{j+1}^{i}}{\Delta R_{i} \Delta z_{j}} R_{i}$

$A_{3} \equiv \frac{\dot{E}_{j+1}^{i+1}-\dot{E}_{j}^{i+1}+\dot{E}_{j}^{i}-\dot{E}_{j+1}^{i}}{\Delta R_{i} \Delta z_{j}}$

With this formula,

$$
\begin{aligned}
L_{j}^{i}= & \pi \Delta R_{i} \Delta z_{j}\left[2 A_{0}\left(R_{i+1}+R_{i}\right)\right. \\
& +\frac{4 A_{1}}{3}\left(R_{i+1}^{2}+R_{i+1} R_{i}+R_{i}^{2}\right) \\
& +A_{2}\left(R_{i+1}+R_{i}\right)\left(z_{j+1}+z_{j}\right) \\
& \left.+\frac{2 A_{3}}{3}\left(R_{i+1}^{2}+R_{i+1} R_{i}+R_{i}^{2}\right)\left(z_{j+1}+z_{j}\right)\right] .
\end{aligned}
$$

Finally, the edge-on surface brightness $\Sigma$ is given by the discretization of Eq. (25) and so, in our scheme,

$\Sigma_{j}^{i}=\sum_{k=i}^{n_{R}-1}\left(\dot{E}_{j}^{k+1}+\dot{E}_{j}^{k}\right)\left(\sqrt{R_{k+1}^{2}-R_{i}^{2}}-\sqrt{R_{k}^{2}-R_{i}^{2}}\right)$. 